Gerichtshilfe im Ermittlungsverfahren umfassender Gebrauch gemacht werden. Das rechtzeitige Erkennen von Auffälligkeiten, Besonderheiten und möglicherweise verhandlungsnotwendigem Verhalten gibt uns Erkenntnisse über einzuleitende Handlungsschritte.

Die Gerichtshilfe befindet sich nach diesen Ausführungen möglicherweise auf halbem Wege, konzeptionell ist sie - mögen auch eine Reihe von Problemen vorhanden sein - den ministeriellen Regelungen etliche Schritte voraus. Die Arbeitsgemeinschaft Deutsche Gerichtshilfe und die Landesarbeitsgemeinschaften haben wiederholt und seit langem auf die Notwendigkeit zur Schaffung verbindlicher Rahmenbedingungen, die auch für die Auftraggeber bindend sein sollten, hingewiesen. In jedem Generalstaatsanwaltschaftsbezirk, in jedem Bundesland können derartige Festschreibungen autonom erfolgen.
- Die weitaus bessere und umfassendere Lösung wäre eine bundeseinheitliche gesetzliche Reform der Strafprozessordnung. Wenn auch nicht allumfassend, so könnte in ganz speziellen Bereichen eine verpflichtende Beiziehung der Gerichtshilfe für eine klarere praktische Umsetzung sorgen.

- Erst die praktizierte Zusammenarbeit im Ermittlungsverfahren bringt Synergieeffekte, Arbeitsvorteile für Staatsanwälte und Richter und somit Kostenersparnis. Deshalb muss die Beiziehung der Gerichtshilfe zu den Standards einer juristischen Arbeit gehören.

- Wir wünschen uns, dass die Entscheidungsträger dieses erkennen und ihren Focus nicht vorwiegend auf Bewährungshilfe richten.

- Wir fordern, an Verfahrensgängen ausgerichtete interdisziplinäre Arbeits- und Lösungsansätze umfassend zu erproben.
- Die weitere Entwicklung der sozialen Strafrechtspflege soll generell die vernetzte $\mathrm{Zu}$ sammenarbeit vorsehen.

- Eine Alternative zur Bündelung fachlicher Kompetenz durch interdisziplinäre Zusammenarbeit sehen wir nicht.

- Mit unserem Beitrag wollen wir eine ganze Reihe von verbindlichen Festschreibungen, die sich sowohl an die Juristen, Sozialarbeiter wie auch an die Politik wenden, einfordern.

- Falls unsere Ausführungen auf Widerspruch stoßen, erbitten wir kritische Äußerungen gepaart mit praktikablen Gegenvorschlägen.

In diesem Sinne.

Ute Seidler ist Gerichthelferin in Lübeck und zusammen mit Rainer-Dieter Hering aus Tübingen im Präsidium der AG Deutsche Gerichtshilfe, Kontakt: r.d.hering@t-online.de

\title{
Sozialpsychologische Überlegungen zur Anfälligkeit von Justizvollzugsbediensteten für »mafiöse Verstrickungen«
}

Michael Alex

$\mathrm{N}$ un hat auch Sachsen-Anhalt seinen Justizvollzugsskandal! Nachdem zuletzt in Brandenburg im Frühjahr 2004 in mehreren Justizvollzugsanstalten des Landes in größerem Umfang Bedienstete sich Vorteile erschlichen hatten oder in mafiöse Strukturen eingebunden waren, stürmten am 12.12.2004 ca. 500 Polizisten - weitgehend aus anderen Bundesländern - das unter dem Namen »Roter Ochse« bekannte Gefängnis in Halle/Saale, stellten u.a. 12 Handys, illegale Drogen und Anabolika sicher und nahmen zahlreiche Akten mit »brisanten Informationen ", die von Bediensteten in die Zellen geschmuggelt worden sein sollen, mit (Mitteldeutsche Zeitung (MZ) vom 13. und 14.12.2004). 8 Bedienstete wurden aufgrund von seit fast einem Jahr geführten Ermittlungen verdächtigt, mit Gefangenen aus der sog. OK gemeinsame Sache gemacht zu haben, gegen eine Beamtin wurde sogleich Haftbefehl erlassen.

Es folgte die in solchen Fällen übliche staatliche Reaktion. »Zur Gefahrenabwehr" wurden an den folgenden zwei Tagen alle Mitarbeiter in den Gefängnissen des Landes beim Betreten und Verlassen der Anstalten durchsucht, wobei der Justizminister diese Maßnahme selbstver- ständlich nicht als Ausdruck eines Generalverdachts verstanden wissen wollte. Der Vorsitzende des Hauptpersonalrats sah sich in einem Interview bemüßigt darauf hinzuweisen, dass nur 8 von 1.000 Mitarbeitern verdächtigt würden, "mit den schweren Jungs « gemeinsame Sache gemacht zu haben (MZ vom 14.12.2004). Und wie meist in solchen Fällen wurde umgehend »eine Kommission von bundesweit tätigen Experten« berufen, die bis Ende März 2005 die Zustände in allen Gefängnissen des Landes untersuchen soll (MZ vom 29.12.2004). "Die Justiz geht unerbittlich gegen schwarze Schafe in den eigenen Reihen vor «, so der Justizminister des Landes Sachsen-Anhalt laut MZ vom 14.12.2004. Auch zusätzlich ergriffene Maßnahmen sind in diesem Kontext der »Zerschlagung eines Netzwerks « zu sehen, so die geplante Rotation aller Beamter in der betroffenen JVA alle drei Monate, um einer engen Verbindung von Gefangenen und Aufsichtspersonal und damit der »Erpressbarkeit« vorzubeugen.

Es gehört nicht viel Phantasie dazu, das Ergebnis der Untersuchungen der Vorfälle vorauszusagen. Die Feststellungen werden darauf hinauslaufen, dass es sich bei den vom Dienst suspendierten Bediensteten um Ich-schwache
Persönlichkeiten gehandelt habe, die nicht die erforderliche Distanz zu den ihnen anvertrauten Straftätern gehalten hätten, ein Aspekt, dem künftig bei der Ausbildung noch mehr Aufmerksamkeit als bisher gewidmet werden müsse. Damit ist die Angelegenheit dann vollends individualisiert.

Doch das dürfte nur die halbe Wahrheit sein. Man kann erfahrungsgemäß davon ausgehen, dass durch die Razzia nur die Spitze eines Eisbergs entdeckt worden ist, und vieles spricht dafür, dass die Ursachen für die Ereignisse nicht nur in der Psyche einzelner Beamter zu suchen sind, sondern in der Struktur des Systems Justizvollzug.

Seit Jahrzehnten ist der Strafvollzug erfolglos bemüht, das Image seiner Mitarbeiter in der Öffentlichkeit zu verbessern, indem beispielsweise die besondere Bedeutung des »Allgemeinen Vollzugsdienstes « bei der »Resozialisierung « herausgestellt wird. Dennoch halten sich in der Öffentlichkeit hartnäckig Berufsbezeichnungen wie »Wärter « oder »Aufseher «, so auch in der Berichterstattung über den aktuellen Skandal. Wieso erfährt die Tätigkeit im Strafvollzug in der Gesellschaft nicht die Anerkennung, die in 
Broschüren, mit denen Justizministerien um Anwärter für eine Tätigkeit im Justizvollzug werben, nahe gelegt wird? Ist es denkbar, dass die tatsächliche Rolle der Vollzugsbeamten doch ein wenig von dem Ideal abweicht, wonach sie »durch ihren ständigen Kontakt mit den Gefangenen auf diese einen starken Einfluss ausüben und die Atmosphäre in einer Anstalt entscheidend prägen « (Justizministerium des Landes Nordrhein-Westfalen 1992, 13)? Noch deutlicher wird das Dilemma in einer Werbebroschüre aus Brandenburg (Ministerium der Justiz des Landes Brandenburg 1993, 3-5) mit dem Untertitel »Ihre berufliche Zukunft? «, in der zu Beginn vermeintlich völlig irrige Vorstellungen über die Tätigkeit im Strafvollzug aufgelistet werden:

«Mal ehrlich, vielleicht denken Sie auch:

1. Die Wärter haben doch nichts anderes zu tun, als den ganzen Tag Türen auf- und zuzuschließen; wie langweilig!

2. Die Schließer haben ja doch nichts zu sagen und von den Gefangenen werden sie auch nicht für voll genommen!

3. Nur mit Schwerverbrechern zu tun zu haben - das färbt ab!

4. Das ist ein Beruf für Bekloppte - das kann doch jeder!

5. Die verdienen schlecht - und mit den Aufstiegsmöglichkeiten sieht's auch ganz bescheiden aus!

Also: Im Knast arbeiten - um Gottes willen, das wäre das Letzte!«

Der Text fährt fort: »Lesen Sie mal weiter ... Sie werden sich wundern!

Zugegeben: Im geschlossenen Vollzug haben Sie die Haftraumtüren auf- und zuzuschließen: wenn die Gefangenen die Mahlzeiten bekommen, wenn sie zur Arbeit, zum Aufenthalt im Freien, zum Fernsehen, zum Sport gehen. Das ist gar nicht wenig. Wenn Sie aber denken, das ist alles, haben Sie sich ganz schön getäuscht.

Wir erwarten nämlich, dass Sie mit den Gefangenen sprechen: nicht nur übers Wetter und das letzte Fußballspiel im Fernsehen; sondern auch darüber, wo den Gefangenen der Schuh drückt. Sie sollen den Gefangenen kennen und wissen, warum er in der Freiheit nicht zurechtgekommen ist, wer zu Besuch kommt und wer nicht, was ihn nach der Entlassung erwartet und wo Hilfe zur Selbsthilfe ansetzen kann.

Das Ganze nennt man Behandlung, im Jugendvollzug Erziehung. ..."

Diese Darstellung der Vollzugswirklichkeit ist so platt, wie sie sich liest! Bekanntlich ist die Verquickung von Geschäfts- und »Unterwelt« oder auch von Verwaltungsbürokratie und »Milieu« in unserer Gesellschaft kein ganz seltenes Phäno- men und der Strafvollzug ist keine Insel der Glückseligen. Im Gegenteil, er ist dem Einfluss Mächtiger - gleich welcher Couleur - in besonderem Maße unterworfen. Denn Justizvollzug ist vor allem geprägt durch »Macht", Macht von Bediensteten gegenüber Gefangenen, Macht von Vorgesetzten gegenüber Untergebenen, Macht von Aufsichtsbehörden gegenüber Anstaltsleitungen etc. Noch immer wird diese Macht gern in Form von Disziplinarmaßnahmen durchgesetzt, Disziplinarmaßnahmen gegen Gefangene, Disziplinarmaßnahmen gegen Mitarbeiterinnen und Mitarbeiter. In Fortbildungsveranstaltungen und Fachzeitschriften wird zwar immer der Eindruck vermittelt, als seien "Zielvereinbarungen « und »Neue Steuerungsmodelle« längst Standard im Justizvollzug, ist der eher triste Alltag in vielen Anstalten auch 30 Jahre nach Inkrafttreten des Strafvollzugsgesetzes in der Regel immer noch (oder wieder?) wenig verändert. Der Strafvollzug ist mancherorts weiterhin eher "paramilitärisch « organisiert (vgl. Rehn, Hamburger Morgenpost vom 22.06.2004) mit einer streng hierarchischen Struktur. Ganz unten sind die Gefangenen, die in einer weltfremden Versorgungsstruktur mit Hospitalisierungseffekten untergebracht sind. Darüber stehen die Mitarbeiter des Allgemeinen Vollzugsdienstes mit einer Vielzahl wenig verantwortungsvoller sog. Verfügungstätigkeiten, die wenig mit Behandlung zu tun haben, sondern ausschließlich der Aufrechterhaltung eines bürokratisierten und abgeschotteten Tagesablaufs dienen (vgl. zu den Gefahren für das interaktive Miteinander durch neue Anforderungen Rehn 2003, 68). Erst oberhalb dieser Ebene beginnen Tätigkeiten, die eine gewisse Selbständigkeit und Gestaltungsfreiheit eröffnen.

Eine solche Struktur lädt zu Rollenunsicherheit und künstlicher Abgrenzung gegenüber dem Klientel ebenso ein wie zu unprofessioneller Spaltung auf der Grundlage von Sympathie bis hin zum Wechsel der Fronten. Die Nähe zum Gefangenen und die relativ ähnliche Ohnmacht in der Gesamtorganisation lassen sich für viele Bedienstete nur ertragen, wenn sie sich extrem von den Gefangenen abgrenzen, die man meint, abwerten und in die Schranken weisen zu müssen. Jedes für die langfristige Perspektive noch so unbedeutende Verhalten im Vollzug wird beobachtet und beurteilt und soll möglichst mit Mitteln primitivster Konditionierung abgestellt werden. Der Toleranzspielraum sinkt auf ein Mindestmaß, erst recht, wenn ein Aufbegehren gegen die totale Institution wahrgenommen wird, das etwa in häufigen Beschwerden zum Ausdruck kommt. Schwächere Gefangene sind den auf sehr subtiler Ebene ablaufenden Abgrenzungsprozessen hilflos ausgeliefert und lassen sich widerstandlos demütigen. Ganz anders erfahrene Gefangene, die auch außerhalb des Justizvollzuges gelernt haben, die Ängstlichkeit in subalternen Strukturen für ihren Vorteil zu nutzen. Der Strafvollzug in besonders rigiden Einrichtungen ist für sie ein besonders geeignetes Betätigungsfeld, zumal durch Vorenthal- ten selbst elementarster »Genüsse « wie rohen Eiern, Frischfleisch oder Backzutaten ein Umgehen der Vorschriften in der Hausordnung geradezu provoziert wird. Mag das Selbstbewusstsein dieser Gefangenen auch nur die Fassade ihrer Rolle im subkulturellen Milieu sein, so machen sie durch ihr Auftreten doch deutlich, dass sie sich von der »Spießerwelt « des bürgerlichen Beamtenapparates nicht einschüchtern lassen, und verkörpern eine vermeintlich zufriedenstellendere Gegenkultur. All das, was der »loyale« Beamte an Begrenzung seiner Entfaltungsmöglichkeiten duldet, ist für sie kein Hindernis, sondern wird als Herausforderung erlebt. Dass eine solche Missachtung gesellschaftlicher Normen für jemanden, der in diesen Grenzen eingezwängt ist, nicht in jedem Fall Abwehr hervorruft, sondern auch einen faszinierenden Aspekt haben kann, liegt auf der Hand. Dann braucht es nicht mehr einen behutsamen Aufbau der Erpressbarkeit, sondern es kommt zur Identifikation mit der durchsetzungsfähigen Subkultur. Will man Justizvollzugsbedienstete gegenüber solchen Identifikationsprozessen immunisieren, muss man ihr Selbstbewusstsein stärken. Das aber kann nur geschehen, wenn Hierarchien abgebaut werden, Vertrauen in die Fähigkeiten der Mitarbeiter entwickelt wird und Gestaltungsmöglichkeiten ausgebaut werden. Erst wenn sich Beschäftigte mit ihrer Arbeit identifizieren können, verlieren alternative Lebensentwürfe an Attraktivität. Solange die Unzufriedenheit mit den Arbeitsbedingungen so groß ist, wie man für die betroffenen Anstalten vermuten kann, wird es immer wieder zum »Rollenwechsel « kommen, weil es den »Herrschern« im subkulturellen Milieu gelingen wird, die extremen Abhängigkeiten und Rollenunsicherheiten eines Teils des Personals geschickt für ihre Ziele zu nutzen. Selbst schlichte Freundlichkeit gegenüber Gefangenen wird im Strafvollzug von KollegInnen und Vorgesetzten häufig schon argwöhnisch betrachtet, wodurch ebenfalls Ablösungsprozesse von der Organisation gefördert werden. Die Probleme liegen also nicht in erster Linie in der Ausbildung, sondern in einer Praxis, die nicht selten $\mathrm{zu}$ einer »regressiven Entprofessionalisierung « (vgl. Schmidbauer 2004, 175 ff.) führen kann. Hier ist vor allem ein anderer Führungsstil als die traditionelle Verwaltungshierarchie gefragt!

\section{Literatur:}

Justizministerium des Landes Nordrhein-Westfalen (1992): Strafvollzug in Nordrhein-Westfalen, 9. Aufl., Düsseldorf

Ministerium der Justiz des Landes Brandenburg (1993): Der Allgemeine Vollzugsdienst, Potsdam

Rehn, G. (2003): Sozialtherapie - Anspruch und Wirklichkeit 2003, in: Neue Kriminalpolitik. 15. Jg. Heft 2, 66-69

Schmidbauer, W. (2004): Persönlichkeit und Menschenführung, 2. Aufl., München

Der Autor ist Leiter der offenen Abteilung der sozialtherapeutischen Anstalt Halle 